\title{
Cultural Competence and the Global Role of Dietitians: A Haitian Medical Mission and Inter-Professional, Service-Learning Nutrition Course
}

\author{
Joanne Christaldi ${ }^{*}, 1$ and Jessica R. Bodzio ${ }^{2}$ \\ ${ }^{1}$ West Chester University of Pennsylvania, 309 Sturzebecker Health Science Center, West Chester, PA 19383, USA \\ ${ }^{2}$ Department of Nutrition and Dietetics, Marywood University, 2300 Adams Avenue, Scranton, PA 18509, USA
}

\begin{abstract}
As the poorest Western Hemisphere country, Haiti has a long history of health disparity including elevated rates of hypertension, HIV/AIDS, and tuberculosis. In the summer of 2012, a four-week knowledge and skills based course, including a one-week medical mission, was developed to meet the need for medical care in Haiti, and to provide an interprofessional, service-learning environment to enhance students' cultural competence. A nonprofit volunteer organization that connects healthcare people with a community in need assisted in developing the mission trip. Background on the culture of Haiti, medical education, and development of nutrition education materials were incorporated into the course. Students participated in classroom activities, assisted with development of nutrition education materials, and maintained a reflective journal during the mission trip. Basic nutrition education, nutrition assessment and evaluation, and breastfeeding instruction were provided to Haitian patients. Additionally, students and faculty interacted with each member of the healthcare team; facilitating a greater understanding of an interprofessional approach to medical care. Incorporating a medical mission trip into dietetics education provides an opportunity to increase cultural competence of faculty and students while providing another outlet for students to gain hands-on experience. Interprofessional service-learning further enhances the educational experience and should be considered as a worthwhile educational technique. Programs looking to incorporate such a mission trip into their curriculum can follow a similar scheme of course development.
\end{abstract}

Keywords: Dietetic students, service learning, cultural competence, Haiti.

\section{INTRODUCTION}

As the poorest country in the Western Hemisphere, Haiti has a long history of health disparity. Rates of hypertension, HIV/AIDS, and tuberculosis are eminent and these diseases require meticulous care and continued patient follow-up [1]. Often times, Haitian clinics rely on volunteers to provide this necessary care. Cultural competence is a set of behaviors, attitudes, and policies that are performed to enable effective work in cross-cultural and global situations and is a necessary factor for decreasing disparities in health care [2]. Cultural competence of members of the Academy of Nutrition and Dietetics has been identified as a strategy for improving the health of Americans [3]. "Service-learning is a teaching and learning strategy that integrates meaningful community service with instruction and reflection to enrich the learning experience, teach civic responsibility, and strengthen communities" [4]. "Interprofessional education occurs when students from two or more professions learn about, from and with each other to enable effective collaboration and improve health outcomes". Interprofes-

\footnotetext{
*Address correspondence to this author at the West Chester University of Pennsylvania, 309 Sturzebecker Health Science Center, West Chester, PA 19383; Tel: 610-436-1038; Fax: 610-436-2860; E-mail: jchristaldi@wcupa.edu
}

sional education is essential in developing practitioners who can effectively function in the collaborative health care environment, which can lead to improved health outcomes and optimal use of resources [5].

In the past, medical, nursing, and other allied health students have had opportunities to travel abroad to provide health care in developing countries. Given that nutrition and diet greatly affect many of the health disparities in Haiti, it is essential to provide nutrition assessment and education to these individuals. These factors prompted a one-week medical mission trip in the summer of 2012. Four nutrition faculty and students took part in an interprofessional medical mission to Haiti. The medical mission was coordinated by a nonprofit organization and the nutrition faculty and students were the first nutrition professionals to volunteer with this nonprofit organization in Haiti. The nutrition faculty and students were required to take on the sizeable tasks of course development and research on appropriate nutrition assessment and education materials that would be needed. The purpose of this paper is to provide information for developing cultural competence in dietitians and nutrition students through a service-learning, interprofessional medical mission in Haiti and preparatory didactic course. Information related to the steps needed to design, implement, and evaluate the course is also included. Furthermore, the 
course development and structure can serve as a model for other medical and allied health educational programs. Having a stronger cultural awareness and increased cultural knowledge can improve dietitians' and dietetic students' cultural competence while interprofessional education can improve their ability to effectively function as part of a collaborative healthcare team. The medical mission and course were for educational and service purposes only and therefore this project was exempt of Institutional Review Board approval.

\section{THEORETICAL FOUNDATION}

One theoretical model used for this project was The Process of Cultural Competence in the Delivery of Healthcare Systems by Josepha Campinha-Bacote [6]. This model defines how healthcare professionals can effectively work within the cultural context of a patient/client and is a process of becoming, not already being, culturally competent. There are five interrelated constructs of the model - cultural awareness, cultural knowledge, cultural skill, cultural encounters and cultural desires. All five of the constructs helped to inform the course development and planning of the medical mission trip. The five constructs were used in the following ways: in the student application process for taking part in the course and medical mission (cultural desires), the nutrition course development (cultural awareness and cultural knowledge) and the medical mission (cultural skill and cultural encounters).

A second theoretical model used for this project was Kolb's Experiential Learning Model [7]. This model suggests that learning occurs when individuals understand experiences and transfers that understanding into knowledge. Additionally, learning is a continuous process informed by experience and involves interactions between the individual and the environment. Kolb suggests that in order for learning to occur, individuals must go through four processes: concrete experience, reflective observation, abstract conceptualization and active experimentation. Because service learning is a form of experiential learning, this concept was the foundation for both the dietetics course development and the planning of the medical mission. In order for students to effectively learn about healthcare, it is essential to incorporate both the didactic knowledge and the hands-on experience.

Interprofessional education, "when students from two or more professions learn about, from and with each other to enable effective collaboration and improve health outcomes" was the pedagogical approach used to create more adaptable, collaborative, practice-ready health care workers who can better meet client needs [5]. The four main competency domains for interprofessional collaborative practice include: value/ethics for interprofessional practice, roles/ responsibilities, interprofessional communication, and teams and teamwork [8]. This interprofessional medical mission allowed students to recognize the value of other health care team members, facilitated discussion about and observation of the roles and responsibilities of each member, fostered communication among team members, and enhanced each member's knowledge of teams and teamwork.

\section{COURSE AND MEDICAL MISSION DEVELOPMENT}

Strengthening the cultural competence of nutrition faculty and students and enhancing the student's education through experiential/service-learning with other health care professionals were the primary motivating factors for the development of an interprofessional education (IPE) course entitled: Interdisciplinary Assessment, Education, and Service in Haiti. This innovative course was developed with a focus on the pedagogical approaches of service-learning and IPE. Faculty members in the Nutrition and Dietetics, Physician Assistant (PA), and Nursing (RN) programs within the College of Health and Human Services (HHS) researched the need for medical service in developing countries. This investigation revealed a significant demand for medical care in Haiti. The faculty determined that a one-week medical mission trip to Haiti, with a corresponding knowledge and skills based course, should be developed to not only meet the remarkable need for medical care in Haiti, but also to provide the opportunity for an interprofessional, servicelearning environment that would enhance students' knowledge of the roles of other healthcare team members while also enhancing their cultural competence and their understanding of the role of the dietitian in a global setting.

A nonprofit volunteer organization that connects healthcare professionals and resources with a community in need was chosen by the interprofessional team and assisted in developing the mission trip itinerary. Housing at the volunteer headquarters in Haiti was limited and restricted the number of team members from nutrition to two faculty and two students. The two nutrition faculty team members volunteered while the two nutrition student positions were determined through an application process. Nine nutrition students submitted formal applications for the two open positions for this inaugural mission trip. The application process included a formal interview, letter of application and recommendation letters. Additional considerations included if the student could speak French or Haitian Creole, completion of an introductory medical nutrition therapy course or experience with nutrition assessment and education, and excellent heath and maturity level. This process allowed the faculty to select individuals who would exhibit flexibility, leadership, and respect during interactions with the patients at the clinics in Haiti, the other team members, and the permanent healthcare workers at the clinics. The application process also allowed faculty to determine who had critically contemplated their motivation for applying to participate in the mission and who had considered the realties associated with the trip.

\section{COURSE DESCRIPTION AND EXPERIENCE}

The Interdisciplinary Assessment, Education, and Service in Haiti course was developed to meet the following four objectives:

- To develop and apply clinical screening, assessment, intervention, and nutrition education skills.

- To encourage mentorship, teamwork, and collaboration among an interprofessional healthcare team.

- To cultivate specialized skills and experiences that foster a learning environment. 
- To develop a deeper understanding of cultural differences and how they relate to the health status of a distinctive population.

The faculty, each with clinical experience in their respective discipline, worked closely to design the course from an interprofessional perspective. Representatives from the nonprofit organization also provided literature on the most prevalent diseases and conditions encountered at their clinics and a formulary for prescription medications most commonly ordered. This information was incorporated into the course content. Additionally, experts in other areas were invited as guest lecturers to further enhance the learning experience, to better prepare the students for the mission trip, and to guide participants through the reentry process. The course schedule was created to allow for ample classroom time prior to the medical mission as well as after returning to campus. The team met for four, three hour classes prior to travel and twice after returning. This was a three-credit, elective course facilitated by faculty from the Nutrition and Dietetics, PA, and RN programs.

The course offered an overview of diseases/conditions prevalent in Haiti. Topics included:

- Cholera

- Dehydration

- Scabies and other skin conditions/infections

- Malaria

- HIV/AIDS

An interprofessional approach to clinical screenings, individualized assessments, and culturally sensitive interventions was emphasized. The environment of the classroom fostered teamwork and collaboration among disciplines as well as between students and faculty. This collaboration continued throughout the medical mission where faculty members were able to individually mentor each student.

Additionally, the course offered exposure to the Haitian culture and enhanced the student's cultural competence in preparation for their medical mission in Haiti. Experts in areas related to the Haitian culture, in service-learning, and in international travel were invited as guest lecturers. These guests included an individual who had visited Haiti twice within the two years prior to the commencement of the medical mission and offered invaluable first-hand knowledge of the Haitian culture; the HHS Dean who prepared students to be culturally sensitive and to treat all people with respect and compassion; the Associate Director of the International Affairs office who spoke on the logistics of the trip (i.e., flight itinerary, insurance, etc.); and the director of the University's Counseling and Student Development Center who provided a debriefing session to the team members after returning from Haiti.

No formal textbooks were required for this course, however two cultural books were assigned and discussed in class. One book was written by a woman who had a life changing experience as a Haitian medical volunteer. She completed interviews and wrote the book to provide information about post-earthquake Haiti from a Haitian perspective. The second book was written by a woman who has extensive cultural experience. She works in the field of leadership and personal development. The book provides tremendous insight into understanding other cultures.

\section{COURSE ASSIGNMENTS}

The course requirements included assignments that were completed prior to, during, and after the mission trip. Attendance and participation in all interprofessional classroom, preparatory, and post-trip activities was expected. As a partnership, and with the guidance of faculty, the nutrition students created educational materials pertaining to the basics of overall good nutrition, maintained reflective journals during the mission trip, photographed their experience, developed a culminating oral presentation, and were asked to provide an evaluation of the overall course and mission trip.

In general, students were extremely positive about the mission trip and course requirements and provided feedback for improved educational materials and counseling skills. Fig. (1) shows a graphical representation of the course assignments.

\section{MEDICAL MISSION DESCRIPTION AND EXPERIENCE}

During the one-week medical mission, the interprofessional team of students and faculty went to five urban and rural health clinics in Haiti. Two primary components of nutrition were provided by the students and faculty: assessment and education. Nutrition assessment included performing height and weight measurements of infants, adolescents, and adults. Many patients had written documentation (i.e., Haitian medical charts) from prior visits to the clinic which included prior body weight measurements, however height measurements were not indicated and the vast majority of patients were not aware of their height. Heights were estimated by standing next to the patients and with the use of portable measuring tapes. Having these anthropometric data allowed for body mass index (BMI) to be calculated. Most patients fell within the "normal weight" BMI classification. However, quality of dietary intake and body composition were not measured. Pediatric patients were weighed on an infant scale and length was assessed with a tape measure. Copies of the Centers for Disease Control and Prevention growth charts [10] were brought and allowed for weight-for-age, length-for-age, and BMI-for-age measurements to be plotted. The growth charts were attached to the patient medical charts with the hope that they will continue to be used at the clinic upon return visits. Additional health history information was provided by the interprofessional team members resulting in a comprehensive assessment of the patient.

The nutrition education component consisted of providing patients with basic dietary information. A large percentage of adult patients had a history of hypertension and presented with elevated blood pressure [1]. These patients were asked about foods commonly consumed to target high sodium choices. Patients were encouraged to limit these foods as much as possible. In addition, patients were instructed to consume higher potassium foods and 


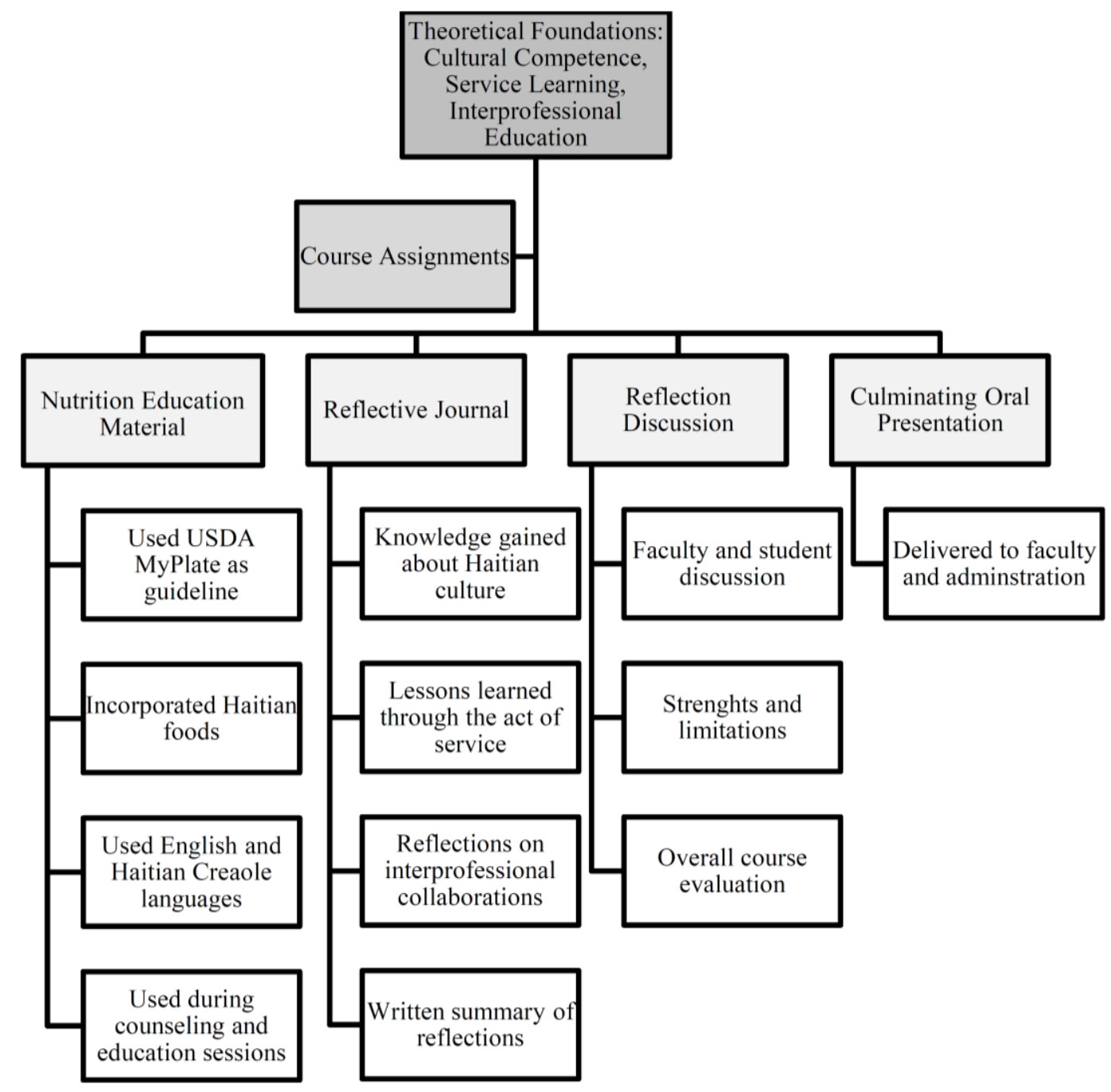

Fig. (1). Graphical representation of course assignments.

emphasis was placed on ensuring proper administration of hypertensive medications. Hypertension was found to be the disease most often requiring nutrition education. Other nutrition education included diabetes and weight management [1]. In addition, several pregnant and lactating women were seen at the clinics. Women were educated on the importance of adequate nutrition during pregnancy and lactation, appropriate scheduling of infant feeding, proper infant positioning, appropriate infant growth patterns, and normal versus unusual infant gastrointestinal habits following breastfeeding.

Nutrition assessment and education were provided by both nutrition faculty and students. Students were observed during assessment and education sessions with guidance and feedback provided by faculty throughout the process at the clinics. Students reflected on the nutrition care they provided and established goals for the following day. Additionally, faculty and students from all disciplines gathered each evening to reflect on the day's events; to share overall insights as well as those pertaining to their interprofessional interactions; to discuss observations about the culture, the people and the community; and how service in this environment was influencing their learning process.
The interprofessional team also interacted with street food vendors and visited a local marketplace during this oneweek mission. These experiences not only enhanced the nutrition education provided by the nutrition team, they also allowed the faculty and students to learn about where Haitians purchase their food. A notable observation during these visits was the high price of foods, especially produce, sold in the marketplace; a clean, enclosed, sanitary environment, as compared to the marginal price of foods sold by street vendors in an environment that is susceptible to food contamination. The knowledge gained from these experiences provided further insight on the Haitian culture and on the probable interaction between food availability and accessibility and disease prevalence in Haiti.

\section{LESSONS LEARNED}

Several lessons were learned throughout this mission trip and course. An overwhelming theme that allowed for a smoother experience was that students and faculty need to be flexible, have a sense of humor, and show compassion. Witnessing the hardships Haitians face every day should not be taken lightly and requires a great deal of compassion. In addition, those involved need to be flexible and open to 
plans changing at the last minute. Furthermore, both nutrition faculty and students should be open to the overall cultural experience. Faculty and students will have an opportunity to learn a tremendous amount about the culture. It is important to study the culture as much as possible before traveling because even seasoned dietitians may struggle with their role in a different cultural environment. Additionally, being culturally competent allows a person to truly serve the people within a community rather than only lend a hand to assist with a situation.

The nutrition team spent many hours prior to travel investigating foods and health conditions in order to create education materials. Handouts were developed that incorporated the USDA MyPlate food guide design [9]. Haitian foods were categorized based on their food group with the idea that they could be distributed to patients. However, these handouts seemed to be more beneficial to the Haitian clinic staff and translators. Nonetheless, the staff and translators can use these materials in the future to demonstrate how foods are grouped together.

Guest speakers were critical to the mission. A few guests spoke to the class prior to travel to discuss the meaning of service-learning, safety and logistics, and their personal experiences in Haiti. These speakers were able to provide a vast amount of insight into the Haitian culture. In addition, their own personal stories contributed to a sense of comfort and clarity to the expectations of Haiti, which provided some peace of mind. Furthermore, having a speaker come to debrief the team upon our return provided an opportunity to discuss how this mission trip has made a substantial impact on our lives.

The team found tremendous benefits in the reflective portion of the service-learning environment. Faculty and students all kept a reflection journal during the mission trip and took part in evening group discussions. This allowed for both private and public expression about the service completed during the day and what was learned about the culture, the interprofessional approach to health care, the role of the dietitian in global health, and ourselves. In addition, the students completed both a reflective paper and culminating oral presentation. This allowed for faculty and members of the community to better understand the servicelearning and impact that this mission trip had on the students.

Finally, given that this was an interprofessional mission trip that included physicians, PAs and RNs, it was extraordinary to have other health disciplines see the role of dietitians in health care. Physician assistant and nursing students were able to observe nutrition assessment and education sessions in addition to recognition of when to consult a dietitian for patient care. These students commented that they were not completely aware of the dietitian's role prior to the mission, which shows a need for incorporating more nutrition students into interprofessional health education. Additionally, students expressed the value of not only working alongside their interprofessional student counterparts, but also witnessing the collaboration among the faculty members.

\section{FUTURE PLANS}

Even though this was the team's first medical mission trip and the first time nutrition professionals volunteered with the nonprofit organization, the overall experience was found to be very rewarding and many aspects of the planning would not be changed. However, it was difficult to determine the kinds and types of nutrition education materials that would be needed for this medical mission. Going forward it was determined that the nutrition team should plan to bring many more growth charts, plan to educate the Haitian clinic staff on their usage, and encourage the inclusion of these charts with the medical record. In addition, development of simple breastfeeding materials to provide to the patients is essential. There appeared to be misconceptions about breastfeeding timing and infant bowel habits which shows a need for nutrition education. In addition, since the Haitian patients did not know their height, it would be beneficial to have a portable stadiometer to more accurately assess this measurement. Finally, given the prevalence of hypertensive patients at the clinics, having more nutrition education materials specific to this condition is greatly needed.

Currently, there is little nutrition education taking place at the medical clinics our teams served in. Therefore, providing brief nutrition education sessions to the clinic staff may help to improve these efforts in the absence of a dietitian. The clinic staff was very receptive to the nutrition education materials provided and patients may benefit from receiving this education. This will also allow for continued nutrition education in the absence of nutrition and dietetics volunteers.

Although enhancing the cultural competence of nutrition faculty and students was one of the primary motivating factors for this medical mission and course, the experience verified that service-learning component of medical mission trips also provide a variety of other benefits to both the patients and students. It is because of this that further investigation on how these interprofessional mission trips can be used to provide nutrition students with supervised practice experience should be a focus going forward. The interprofessional team has decided to continue providing these medical mission trips to students. The course was formally approved by the University's Undergraduate Curriculum Committee to be a permanent part of the curriculum and plans are in progress for continuing medical missions to developing countries. Offering future trips along with an interprofessional course will allow for more students to have a service-learning opportunity in a medical setting and will provide for expanded cultural competence.

Nutrition students generally do not have extensive handson counseling experience. Based on the students' feedback, and in order to increase their confidence and allow them to take on a larger role, counseling vignettes could be developed. These counseling vignettes could be incorporated into the course curriculum and practiced prior to travel.

Finally, the Haitians were very receptive when trying to speak their language and they responded positively even if mistakes were made. Therefore, it would be beneficial to 
spend additional time practicing the language of foods, medical terms and popular sayings prior to travel. This may allow the patients and clinic staff to feel more comfortable during education sessions and may enhance the healthcare provider's ability to serve the patients of these medical clinics.

\section{INSTITUTIONAL MODEL}

The development of this interprofessional medical mission could serve as a model for other healthcare programs looking to provide students with an international service-learning experience. The components of the model fall into six categories:

- Planning

- Student selection

- Course development and components

- Guest lectures pre-travel

- Medical mission trip

- Post-travel components

The planning section includes activities needed to occur first. The selection of a mission organization needs to take place several months in advance since volunteer weeks are
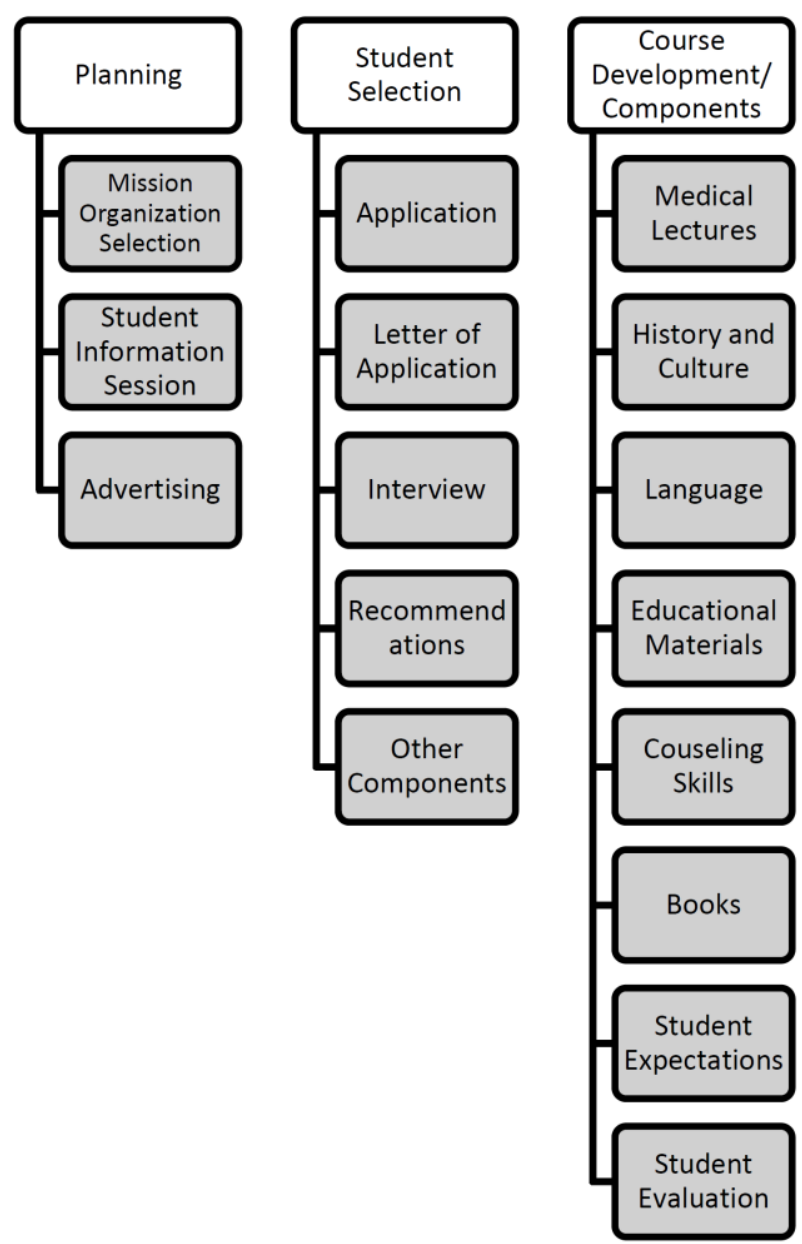

reserved early. Additionally, having an information session for students and advertising the service-learning trip should take place in advance of student selection. The student selection section includes the application and interview process for students. The course development section includes the course components and educational lectures provided prior to travel. In addition, the inclusion of guest speakers should be considered. The medical mission trip includes international travel, self-reflective journal writing and cultural emersion. Finally, post-trip components should include mental health debriefing, student presentation of experiences, and evaluation. Medical and allied health educational programs can transfer this model into their curriculum. The interprofessional team found that this experience was a tremendous success with the inclusion of three disciplines. Including medical professionals such as PAs or medical doctors in addition to RNs should be considered given the nature of the medical mission and the skills that they add to the team. However, varying the types and number of disciplines can be altered as needed. See Fig. (2) for the institutional model.

\section{CONCLUSIONS}

Medical mission trips can be another way to grow the nutrition and dietetics profession. Incorporating a medical mission trip into nutrition education provides an excellent
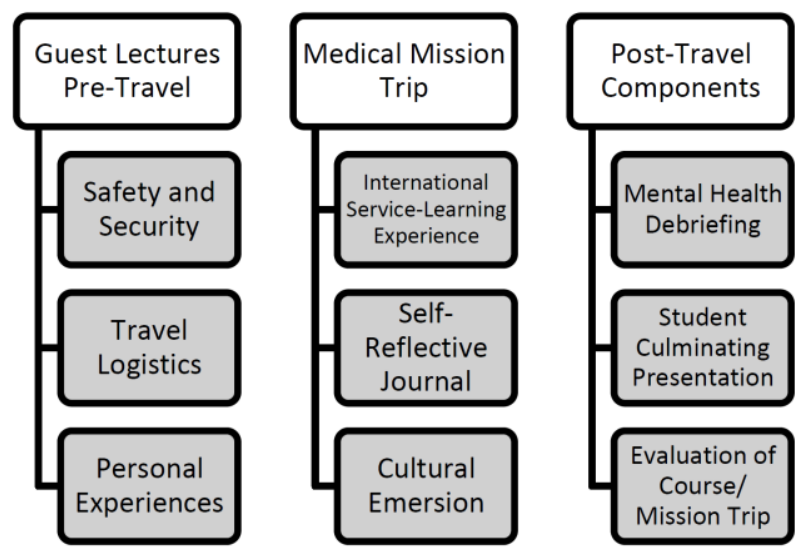

Fig. (2). Institutional model for implementing an interprofessional medical mission and service-learning course. 
opportunity to increase cultural competence of both faculty and students. In addition, it provides another outlet for students to gain hands-on experience in an interprofessional, service-learning environment. Overall, the faculty and students learned an enormous amount about the Haitian culture and healthcare practices and took part in experiences designed to improve cultural awareness, communication, counseling, and social interaction. Interprofessional servicelearning enhances the educational experience and allows for witnessing collaboration amongst the healthcare team and should be considered as a worthwhile educational technique. Medical and allied health programs looking to incorporate such a mission trip into their curriculum can follow a similar model of course and mission trip development.

\section{CONFLICT OF INTEREST}

The authors report no conflict of interest.

\section{ACKNOWLEDGEMENTS}

None declared.

\section{REFERENCES}

\footnotetext{
World Health Organization. Country Health Profile 2012. Available from: http://www.who.int/gho/countries/hti.pdf
}

US Department of Health and Human Services, Office of Minority Health, 2005. What is Cultural Competency? Available from: http://minorityhealth.hhs.gov/templates/browse.aspx?lvl=2\&lvlID= 11

[3] Academy of Nutrition and Dietetics. Strategic Plan 2011-2012. Available from: http://www.eatright.org/About/Content.aspx?$\mathrm{id}=8266$

[4] National Service-Learning Clearinghouse. 2013. What is service learning? Available from: http://www.servicelearning.org/whatservice-learning

[5] World Health Organization, 2010. Framework for action on interprofessional education and collaborative practice. Available from: http://www.who.int/hrh/resources/framework_action/en/

[6] Dreachslin JL, Gilbert MJ, Malone B. Diversity \& cultural competency in health care: A systems approach. San Francisco: Wiley 2013.

[7] Kolb D. Experiential learning: Experiences as the source of learning and development. Englewood Cliffs, NJ: Prentice Hall 1984

[8] Interprofessional Education Collaborative Expert Panel 2011. Core Competencies for Interprofessional Collaborative Practice: Report of an expert panel. Washington, D.C.: Interprofessional Education Collaborative. Available from: http://www.aacn.nche.edu/ education-resources/IPECReport.pdf

[9] US Department of Agriculture, 2012. Choose MyPlate. Available from: http://www.choosemyplate.gov/

[10] The Centers for Disease Control and Prevention, 2010. Growth Charts. Available from: http://www.cdc.gov/growthcharts/ cdc_charts.htm 